\title{
Impact of "race" as a social variable for screening for the risk of non-medical opioid use using nomogram
}

\section{Letter to the Editor}

Cite this article: Yennurajalingam S, Bruera $\mathrm{E}$ (2021). Impact of "race" as a social variable for screening for the risk of non-medical opioid use using nomogram. Palliative and Supportive Care 19, 638-639. https://doi.org/10.1017/ S1478951521001358

Received: 25 June 2021

Revised: 27 July 2021

Accepted: 7 August 2021

Author for correspondence:

Sriram Yennurajalingam,

Department of Palliative Care, Rehabilitation, and Integrative Medicine, Unit 1414,

The University of Texas MD Anderson Cancer Center, 1515 Holcombe Blvd., Houston, TX 77030, USA.

E-mail: syennu@mdanderson.org
Sriram Yennurajalingam, M.D. (1) and Eduardo Bruera, M.D.

Department of Palliative Care, Rehabilitation and Integrative Medicine, The University of Texas MD Anderson Cancer Center, Houston, TX, USA

We thank Jones et al. for their review and comments in response to our article, "The development of a nomogram to determine the frequency of elevated risk for non-medical opioid use [NMOU] in cancer patients" (Yennurajalingam et al., 2021a). In this study, we proposed a practical nomogram to assess the risk of NMOU. The risk factors included in the nomogram were known demographic and clinical factors including gender, race/ethnicity, marital status, morphine equivalent daily dose, positive CAGE score ( $\geq 2 / 4)$, and the Edmonton Symptom Assessment Scale itemsanxiety, depression, and financial distress. A score of $\geq 7$ on the Screener and Opioid Assessment for Patients with the Pain tool was considered the gold standard for predicting the risk of NMOU (Akbik et al., 2006).

Jones et al. suggest that the use of race/ethnicity as a social variable in the nomogram was less clear, and may have potential implication of promoting race-based disparities in opioid and pain care rather than improving care. Our paper emphasized (and we do it once more here) that this nomogram does not in any way modify the prescription of opioids to a patient. As it happens with other nomograms, a wrongful interpretation could lead to less opioid prescription for some of the at-risk groups, including nonwhite patients. Perhaps, it would have been helpful to have included a discussion of the complexity and potential misinterpretations of using race as a risk factor in predicting the risk of NMOU in our paper, but we are grateful for the opportunity to address this in our reply to the letter by Jones et al.

This algorithm is not about treatment decision-making but to screen which patients may need more frequent and intense follow-up of patients at risk. The rationale for inclusion of the demographic and clinical factors in the nomogram was multifold: (a) Lack of screening for NMOU in routine outpatient palliative/supportive clinical care. This has a risk for adverse outcomes, including poor pain control, multiple emergency room visits, or hospitalization (Arthur and Bruera, 2019). (b) Nomograms may facilitate screening all supportive/palliative patients for NMOU and identify at risk patients requiring more intense follow-up by an interdisciplinary team so as to provide optimal use of medications for pain and symptom management, counseling, as well as support to patients and their family (Yennurajalingam et al., $2021 \mathrm{~b}$ ), and (c) the use of various factors in the study were based on prior studies on NMOU, and various epidemiological studies to effectively screen cancer and prognosis (Nam et al., 2009; Shariat et al., 2009; Lippold et al., 2019; Lv et al., 2019; Volkow et al., 2019; Yang et al., 2019; Shah et al., 2021). Additionally, our analysis in this study found that the risk of NMOU was significantly associated with the factors included in the nomogram with a $C$-index was 0.803 . With 500 bootstrap repetitions, the $95 \%$ confidence interval of $C$-index was $(0.783,0.822)$. (d) Finally, recent studies have found that the relative morbidity and mortality from opioid overdose is higher and increasing among nonwhite patients (James and Jordan, 2018; Shiels et al., 2018; Friedman et al., 2021). Race/ethnicity is considered important and required variables in scientific review by IRBs and granting agencies, and studies that on purpose avoid collecting and analyzing such data would be hard to conduct and publish (Geller et al., 2011; Pérez-Stable, 2021).

We also emphasized that the findings in this paper are work in progress (Yennurajalingam et al., 2021a), and we agree with Jones et al. that there is great need for further research to better characterize the risk factors including race based on research, consensus of experts in NMOU, and stakeholders considering the complexity and implication of various psycho-socio-spiritual factors (Yennurajalingam et al., 2021a). Vyas et al. (2020) in their recent opinion paper propose the removal of race/ethnicity from existing and future clinical decision-making algorithms. This is based on a concern that such correction might have detrimental effects on a specific vulnerable group. The elimination of major psycho-socio-spiritual variables independently associated with outcomes would force clinicians to prioritize their care based on largely biomedical variables. These might work for some of the disease-oriented specialties mentioned in the paper by Vyas et al. but certainly would be of limited or no help for person-centered supportive and palliative care. We acknowledge that race/ethnicity as a risk factor can be complex and perhaps our paper could have discussed this more overtly. 
Our longer term goal is to eliminate these disparities, so they will not be any more associated with outcomes. In the current clinical environment, clinicians working in supportive and palliative care are universally understaffed. The purpose of this nomogram is to allow them to deliver more frequent and intense care to those who have a higher risk of a developing NMOU and opioid-use disorder, a devastating and potentially lethal complication of opioid treatment for cancer pain.

\section{References}

Akbik H, Butler SF, Budman SH, et al. (2006) Validation and clinical application of the screener and opioid assessment for patients with pain (SOAPP). Journal of Pain and Symptom Management 32(3), 287-293.

Arthur J and Bruera E (2019) Balancing opioid analgesia with the risk of nonmedical opioid use in patients with cancer. Nature Reviews Clinical Oncology 16(4), 213-226.

Friedman J, Mann NC, Hansen H, et al. (2021) Racial/ethnic, social, and geographic trends in overdose-associated cardiac arrests observed by US emergency medical services during the COVID-19 pandemic. JAMA Psychiatry 78(8), 886-895.

Geller SE, Koch A, Pellettieri B and Carnes M (2011) Inclusion, analysis, and reporting of sex and race/ethnicity in clinical trials: Have we made progress? Journal of Women's Health (Larchmt) 20(3), 315-320.

James K and Jordan A (2018) The opioid crisis in black communities. Journal of Law, Medicine \& Ethics 46(2), 404-421.

Lippold KM, Jones CM, Olsen EO and Giroir BP (2019) Racial/ethnic and age group differences in opioid and synthetic opioid-involved overdose deaths among adults aged $\geq 18$ years in metropolitan areas-United States, 20152017. MMWR Morbidity and Mortality Weekly Report 68(43), 967-973.

Lv X, Yu H, Gao P, et al. (2019) A nomogram for predicting bowel obstruction in preoperative colorectal cancer patients with clinical characteristics. World Journal of Surgical Oncology 17(1), 21.
Nam RK, Zhang WW, Trachtenberg J, et al. (2009) Utility of incorporating genetic variants for the early detection of prostate cancer. Clinical Cancer Research 15(5), 1787.

Pérez-Stable EJ (2021). Communicating the value of race and ethnicity in research. www://www.nih.gov/about-nih/what-wedo/science-health-publictrust/perspectives/science-health-public-trust/communication-value-raceethnicity-research.

Shah A, Polascik TJ, George DJ, et al. (2021) Implementation and impact of a risk-stratified prostate cancer screening algorithm as a clinical decision support tool in a primary care network. Journal of General Internal Medicine 36(1), 92-99.

Shariat SF, Scardino PT and Lilja H (2009) Screening for prostate cancer: an update. The Canadian Journal of Urology 15, 4363-4374.

Shiels MS, Freedman ND, Thomas D and Berrington de Gonzalez A (2018) Trends in U.S. drug overdose deaths in non-hispanic black, hispanic, and non-hispanic white persons, 2000-2015. Annals of Internal Medicine 168 (6), 453-455.

Volkow ND, Jones EB, Einstein EB and Wargo EM (2019) Prevention and treatment of opioid misuse and addiction: a review. JAMA Psychiatry $\mathbf{7 6}$ (2), 208-216.

Vyas DA, Eisenstein LG and Jones DS (2020) Hidden in plain sight-reconsidering the use of race correction in clinical algorithms. New England Journal of Medicine 383(9), 874-882.

Yang J, Tian G, Pan Z, et al. (2019) Nomograms for predicting the survival rate for cervical cancer patients who undergo radiation therapy: a SEER analysis. Future Oncology 15(26), 3033-3045.

Yennurajalingam S, Edwards T, Arthur J, et al. (2021a) The development of a nomogram to determine the frequency of elevated risk for non-medical opioid use in cancer patients. Palliative \& Supportive Care 19(1), 3-10.

Yennurajalingam S, Arthur J, Reddy S, et al. (2021b) Frequency of and factors associated with nonmedical opioid use behavior among patients with cancer receiving opioids for cancer pain. JAMA Oncology 7(3), $404-411$. 